\title{
Prior Mating Experience Modulates the Dispersal of Drosophila in Males More Than in Females
}

\author{
Jasper C. Simon • William B. Dickson • \\ Michael H. Dickinson
}

Received: 19 September 2010/ Accepted: 20 April 2011/Published online: 27 May 2011

(C) The Author(s) 2011. This article is published with open access at Springerlink.com

\begin{abstract}
Cues from both an animal's internal physiological state and its local environment may influence its decision to disperse. However, identifying and quantifying the causative factors underlying the initiation of dispersal is difficult in uncontrolled natural settings. In this study, we automatically monitored the movement of fruit flies and examined the influence of food availability, sex, and reproductive status on their dispersal between laboratory environments. In general, flies with mating experience behave as if they are hungrier than virgin flies, leaving at a greater rate when food is unavailable and staying longer when it is available. Males dispersed at a higher rate and were more active than females when food was unavailable, but tended to stay longer in environments containing food than did females. We found no significant relationship between weight and activity, suggesting the behavioral
\end{abstract}

Edited by Yong-Kyu Kim.

J. C. Simon $(\bowtie)$. W. B. Dickson · M. H. Dickinson

Division of Biology, California Institute of Technology,

Pasadena, CA 91125, USA

e-mail: simon@oxy.edu

Present Address:

J. C. Simon

Department of Biology, Occidental College,

Los Angeles, CA 90041, USA

Present Address:

W. B. Dickson

IO Rodeo Inc., 402B South Marengo Ave.,

Pasadena, CA 91101, USA

Present Address:

M. H. Dickinson

Department of Biology, University of Washington,

Seattle, WA 98195-1800, USA differences between males and females are caused by an intrinsic factor relating to the sex of a fly and not simply its body size. Finally, we observed a significant difference between the dispersal of the natural isolate used throughout this study and the widely-used laboratory strain, Canton-S, and show that the difference cannot be explained by allelic differences in the foraging gene.

Keywords Dispersal - Mating experience - Activity . Sexual dimorphism - Behavior - Natural isolate $\cdot$ Canton-S . Foraging gene $\cdot$ Genetic structure $\cdot$ Drosophila

\section{Introduction}

Rotting vegetable matter with fungal and microbial colonies offers all of the known resources required for the livelihood of Drosophila melanogaster (Throckmorton 1975). A single vegetable rot provides the nutrients needed for the growth and development of both larvae and adults, a source of protein for egg maturation, a site suitable for oviposition, and a location for pupariation. Moreover, staying near a rot increases the potential for finding mates and may grant shelter from predators and at least some adverse environmental conditions (but see (Feder 1997)). Because Drosophila obtain multiple resources from a single location, it would seem logical that they would stay at a resource patch indefinitely, but even under a constant physiological state with constant stimuli from ambient environmental conditions they often disperse. Presumably, cues from the flies' internal and local environment combine to influence the probability that flies disperse rather than remain on a patch (Dethier 1964; Kennedy 1978). The dispersal of Drosophila provides a promising model for studies of decision making because of the extensive literature on this species 
Table 1 Known effects of mating on movement for a sample of arthropods

\begin{tabular}{|c|c|c|c|c|c|}
\hline Order & Species (common name) & Male & Female & Behavior & Reference \\
\hline Acari & $\begin{array}{l}\text { Tetranychus urticae (Twospotted spider } \\
\text { mite) }\end{array}$ & n.r. & $=$ & Dispersal & Suiter and Gould (1992) \\
\hline Coleoptera & $\begin{array}{l}\text { Leptinotarsa decemlineata (Colorado } \\
\text { potato beetle) }\end{array}$ & + & - & Flight & Alyokhin and Ferro (1999) \\
\hline Diptera & Aedes aegypti (Mosquito) & n.r. & - & Flight & Jones (1981) \\
\hline Diptera & Aedes aegypti (Mosquito) & n.r. & 0 & $\begin{array}{l}\text { Post blood meal/ pre oviposition } \\
\text { activity }\end{array}$ & Jones (1981) \\
\hline Diptera & Anopheles balabacensis (Mosquito) & n.r. & + & Flight to blood source; biting & $\begin{array}{l}\text { Iwanaga-Sawabe and } \\
\text { Kanda (1990) }\end{array}$ \\
\hline Diptera & Anopheles gambiae (Mosquito) & n.r. & - & Flight (dusk) & Jones and Gubbins (1978) \\
\hline Diptera & Anopheles gambiae (Mosquito) & n.r. & + & Flight (night) & Jones and Gubbins (1978) \\
\hline Diptera & Anopheles gambiae (Mosquito) & n.r. & - & $\begin{array}{l}\text { Post blood meal/pre oviposition } \\
\text { activity }\end{array}$ & Jones and Gubbins (1978) \\
\hline Diptera & Anopheles stephensi (Mosquito) & n.r. & + & Flight (dusk and evening) & Rowland (1989) \\
\hline Diptera & Anopheles stephensi (Mosquito) & n.r. & 0 & $\begin{array}{l}\text { Post blood meal/ pre oviposition } \\
\text { activity flight }\end{array}$ & Rowland (1989) \\
\hline Diptera & Glossina morsitans (Tsetse fly) & n.r. & + & Post reproductive activity & $\begin{array}{l}\text { Rowcliffe and Finlayson } \\
\text { (1982) }\end{array}$ \\
\hline Diptera & Glossina morsitans (Tsetse fly) & n.r. & - & Pre larvaposition activity & Brady and Gibson (1983) \\
\hline Diptera & Toxotrypana curvicauda (Papaya fruit fly) & n.r. & $=$ & Flight (plume tracking, hovering) & Landolt and Heath (1988) \\
\hline Hemiptera & Oncopeltus fasciatus (Milkweed bug) & - & $?$ & Flight & Dingle (1966) \\
\hline Hemiptera & Lygus lineolaris (Tarnished plant bug) & $=$ & $=$ & Flight & Stewart and Gaylor (1994) \\
\hline Hymenoptera & Eretmocerus eremicus (Wasp) & - & - & Dispersal & Bellamy and Byrne (2001) \\
\hline Hymenoptera & Nasonia vitripennis (Wasp) & n.r. & + & Walking, hopping, and flight & $\begin{array}{l}\text { King (1993), King et al. } \\
\text { (2000) }\end{array}$ \\
\hline Hymenoptera & Trichogramma brassiae (Wasp) & + & - & Flight & Pompanon et al. (1999) \\
\hline Lepidoptera & $\begin{array}{l}\text { Agrotis ipsioln (Black cutworm, adult } \\
\text { moth) }\end{array}$ & $=$ & - & Flight & $\begin{array}{l}\text { Sappington and Showers } \\
\text { (1992) }\end{array}$ \\
\hline Lepidoptera & $\begin{array}{l}\text { Helicoverpa armigera (Bollworm, adult } \\
\text { moth) }\end{array}$ & n.r. & - & Flight & Armes and Cooter (1991) \\
\hline Lepidoptera & $\begin{array}{l}\text { Rhyacionia buoliana (European pine } \\
\text { shoot moth) }\end{array}$ & n.r. & + & Flight initiation & Green (1962) \\
\hline
\end{tabular}

+ Increase, - Decrease, $=$ No change, 0 No movement, ? Inconclusive, n.r. Not reported

(Dobzhansky 1973; Grossfield 1978), and the potential for using the available genetic approaches to examine the underlying physiological mechanisms (Callaway 2005; Luo et al. 2008; Zhang et al. 2007).

In addition to needing food and water, most female insects require a mate and an oviposition site for successful reproduction. The relative priority for food, mates, and egg-laying sites has been shown to depend on both the level of hunger and the mating status of the individual (Barton Browne 1993). Many studies suggest that hungry insects modify their behavior to increase their probability of finding food (see within (Barton Browne 1993)). It is also known that mating status affects the movement of many insects (Johnson 1969), although the polarity of this influence does not appear to follow an obvious trend (see Table 1). After mating, females of some fly species shift their preference from sex pheromones to food odors
(Cornelius et al. 2000; Jang et al. 1998). Mediterranean fruit flies, Ceratits capitata, have been shown to shift their behavioral priority from mating to oviposition (Jang et al. 1999). In Drosophila it has been reported that mated females require more food (Carvalho et al. 2006), shift their preference to a high-protein diet (Ribeiro and Dickson 2010; Vargas et al. 2010), emigrate at a lower rate between chambers containing food (Mikasa 1998), and exhibit a higher level of general activity (Isaac et al. 2010). To our knowledge, the effects of mating on the dispersal behavior of male Drosophila have not been investigated.

The primary goal of this work is to investigate if and to what extent mating experience influences the dispersal of male and female Drosophila from food. Additionally, we considered whether changes in the general locomotor activity of flies is sufficient to explain any effects on their dispersal. To address these questions, we carried out 
studies using a custom-built system of connected chambers and automated counters. The modular nature of this technology allowed us to quantify the movement of large numbers of flies between controlled sensory environments.

\section{Materials and methods}

\section{Animals}

We performed experiments on 3- to 4-day-old adults from four laboratory colonies of the fruit fly, Drosophila melanogaster (Meigen). The first colony descended from 200 wild-caught females. The second colony came from the Canton-S stock of the late Ed Lewis. The third $\left(\right.$ for $\left.^{R}\right)$ and fourth $\left(f^{S} r^{S}\right)$ colonies came from stocks possessing the natural-polymorphic variants of the foraging gene from the laboratory of Marla Sokolowski. We reared, entrained, and tested all flies on a set light-dark cycle (LD16:8). Transitions between light and dark were immediate, with the subjective dawn starting at 07:00 and the subjective dusk at 23:00. We maintained fly stocks at $25^{\circ} \mathrm{C}$ and at a relative humidity of either 30 or $60 \%$ on Lewis medium in standard 250-mL bottles (Lewis 1960).

\section{Animal handling}

Unless otherwise noted, we housed groups of 50 flies in vials (AS-515; Thermo Fisher Scientific, Inc., Waltham, MA, USA), on a 2-mL aliquot of food from a medium (Ralph Greenspan, personal communication) consisting of $30 \mathrm{~mL}$ Karo ${ }^{\circledR}$ dark corn syrup, $15 \mathrm{~g}$ sucrose, $15 \mathrm{~g}$ Torula yeast (Lake States, Wisconsin, USA), $10 \mathrm{~g}$ agar, and $1.0 \mathrm{~L}$ distilled water.

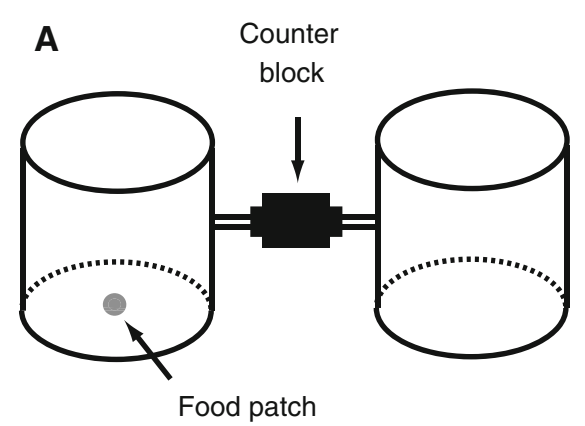

Fig. 1 Instrument devised to study the movement of Drosophila between controlled sensory environments. a Illustration showing two experimental chambers connected by tubes feeding into the opposite sides of a counter block. For dispersal experiments, we either did or did not provide a patch of food in the middle of the chamber floor. b Schematic of a gate and bi-directional counting block. We drove each gate with a solenoid (push-pull type) motor and monitored the
In order to compare mated and virgin flies of a similar age and rearing condition, we collected virgins $<7$ h post-eclosion and divided the collected individuals into three groups: 50 males per vial, 50 females per vial, and a mixture of 25 males and 25 females per vial. To keep housing densities equivalent, 3 days later we combined vials containing the mixture of 25 males and 25 females (providing ample time for them to mate) and then sorted them by sex into two new vials. The result was two vials, the first containing 50 mated males and the second containing 50 mated females. The following day, we tested these mated flies along with the previously collected virgins. To help with counting and sorting, we immobilized flies by cooling them to $4^{\circ} \mathrm{C}$ on a Peltier stage (Marlow Industries, Inc., Dallas, TX, USA).

\section{Experimental test chambers}

We developed a system of hardware and software to help automate studying the movement of flies between controlled sensory environments. The building blocks of this system were opaque, white cylindrical chambers, $9 \mathrm{~cm}$ high and $9.5 \mathrm{~cm}$ in diameter (inner dimensions), which interconnected in a modular fashion (Fig. 1a). Flies placed within a pair of connected chambers moved between adjoining chambers through $5.5-\mathrm{cm}$-long narrow tubes with an inner diameter of $0.55 \mathrm{~cm}$. Tubes from pairs of chambers were connected via a $3.5-\mathrm{cm}$ passageway within an acrylic block that ran through a solenoid-controlled gate and directional IR counter (Fig. 1b). The cross-sectional diameter of this channel narrowed to $0.20 \mathrm{~cm}$. This diameter was large enough to allow a single female to pass through, but small enough to prevent the simultaneous passage of two males.

We designed and built circuit boards with programmable ATmega8 microcontrollers (Atmel, Inc., California,

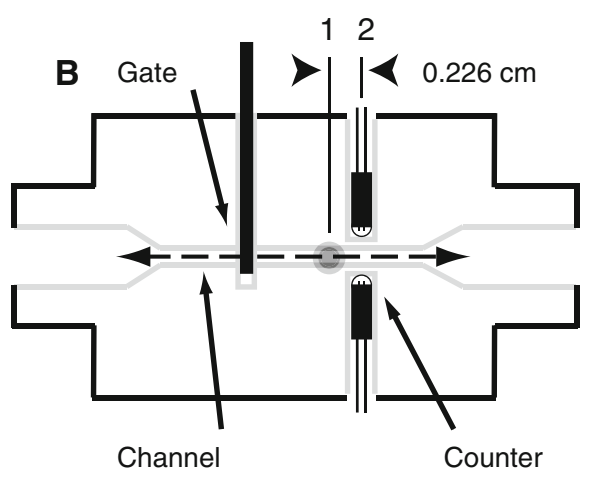

transition of flies through a channel within the counting blocks with two pairs of infrared emitter/detector diodes, denoted as 1 and 2 . Note that the second pair of diodes is offset from the first pair by $0.226 \mathrm{~cm}$ (measured between diode centers) and are not shown in the drawing. The second set of diodes would project normal to the plane of the drawing (gray dot) 
A

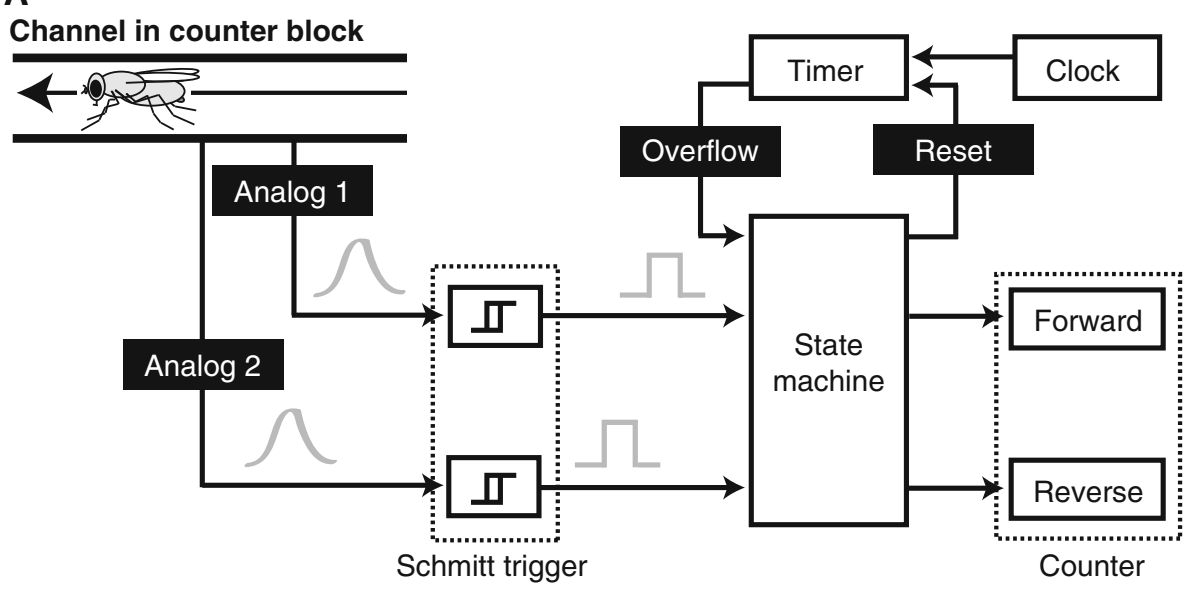

B

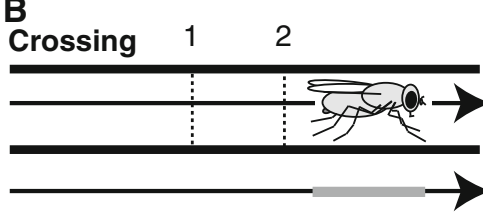

E

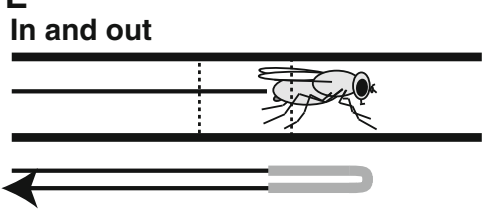

G

Nose poke

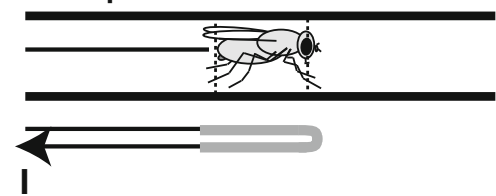

Nose pokes

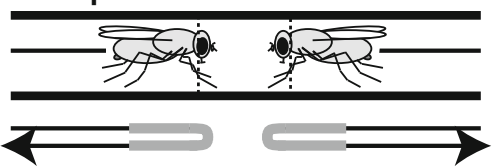

C

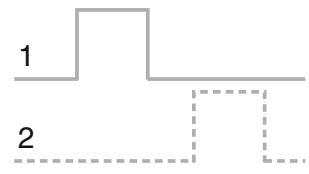

D
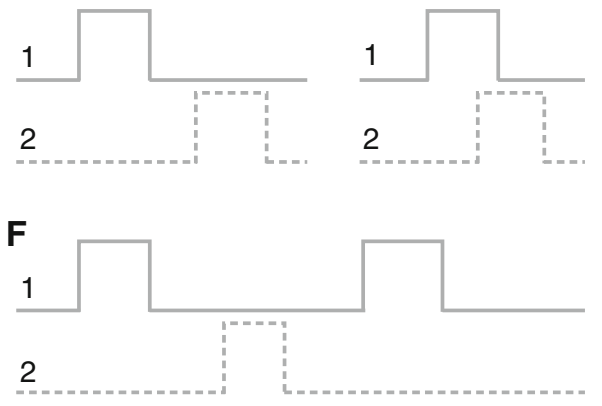

H

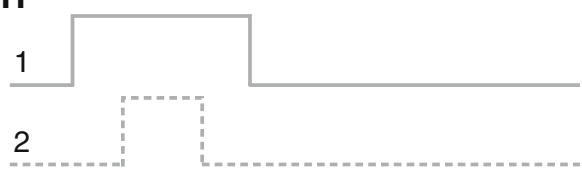

J

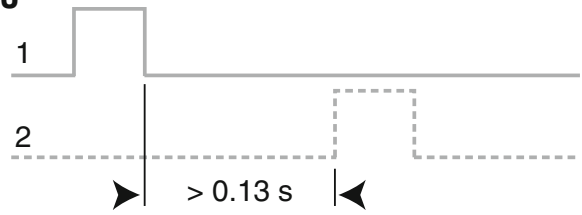

USA) to control the gates and monitor the movement of flies passing through the detectors. Figure 2 illustrates the operational logic of the detector system. We configured a computer to control the opening or closing of the gates and to detect when flies passed though the detectors. Each local microcontroller stored crossing events at a rate of $100 \mathrm{kHz}$. The number of crossing events were stored locally by each counter circuit and subsequently queried by the main computer at a lower rate. For typical experiments using an array of 16 pairs of chambers, we downloaded the accumulated count values to the computer every $26 \mathrm{~s}$.

To test the accuracy of our counters, we introduced groups of 50 flies to the first of two connected chambers, each containing only water, and monitored their movement between chambers for $30 \mathrm{~h}$. At the end of this test, we counted the number of flies observed in the second chamber and compared this number to the number of flies as determined by our automated system. On two separate occasions, we tested each of the 16 counters. The average accumulated errors after $30 \mathrm{~h}$ were 7.2 and $6.7 \%$, with $80 \%$ of these errors being undercounts. To minimize the interference of this counter error in our experiments, we report rates of dispersal, a measure that is more resilient to the accumulation of error over time than absolute counts of flies.

The entire array of chambers sat undisturbed within a temperature-, light-, and humidity-controlled room. Average light levels inside chambers were around 360 lux. (See 
4 Fig. 2 Diagram of the operational logic and examples of behavior near the counter. a Block diagram illustrating how the counter detects and assigns the bi-directional movement of flies. As a fly walks through the channel, it triggers two pairs of emitter/detector diodes, each pair producing an analog signal, denoted as Analog 1 and Analog 2. We used Schmitt triggers to create an all-or-none pulse based on a threshold of these analog signals. The coincidence of these two inputs and a time input pass into a state machine. In order to avoid registering false crossing events, if no additional signal from either of the emitter/ detector pairs reached the state machine within $0.13 \mathrm{~s}$ after a previous signal, then the progression towards registering a crossing event was stopped and the timer was reset. This window of time between signals captured true crossing events, but minimized false signals from two flies approaching the counter from opposing directions (see $\mathbf{i}, \mathbf{j}$ below). A counter tallied the forward and reverse crossing events registered from the state machine. b Illustration showing a typical crossing event. A fly moves through the channel from left to right, first triggering emitter/detector diode 1 and then 2. c, d Corresponding signals from a typical crossing event passing into the state machine. A specific pair of signals may pass into the state machine independently (as shown in c) or together (as shown in d), depending on the size and angle of the fly and the intensity/sensitivity of the emitter/detector diodes. The state machine registered crossings of flies from the opposite direction, from 2 to 1 , similarly. e Illustration of the shortest movement of a fly along the channel that registers a crossing event. A fly moves through the detector far enough to completely pass through and trigger 1 , but only transiently triggers 2 before reversing its direction and returning to where it originated. f Corresponding signals from 'in and out' that register a crossing event. $\mathbf{g}$ Illustration of the farthest movement of a fly along the channel that does not register a crossing event. A fly moves through the detector far enough to trigger a continuous signal in 1 , but triggers only a transient signal in 2 . h Corresponding signals from 'nose poke' that do not register a crossing event. i Illustration of two flies coming from opposite sides, both flies triggering the emitter/ detector diode that they pass through coming from their respective directions before they reverse their direction and return to where they originated. $\mathbf{j}$ Corresponding signals from the combination of two flies approaching from adjacent chambers

Table 2 for the measures of temperature and humidity from specific experiments.) We configured the specific protocols of each experiment (e.g., when or which door opens and closes) and collected data with custom software written in
$\mathrm{C}$ and Matlab (Mathworks Natick, MA, USA). We designed this system to be flexible so that it would be possible to reconfigure the experimental chambers for further studies.

\section{Dispersal assay}

In each set of trials, we introduced single flies or groups of 50 individuals into the first of two connected chambers. Here it is worth noting that we did not observe an abrupt increase in dispersal at a critical threshold density, a "mass migration," as reported previously (Sakai et al. 1958), but rather observed that dispersal scales with density; the fraction of individuals dispersing over a given period of time was comparable for groups over the range of $<10$ to 1000 individuals (Simon 2010). In all experiments we deprived flies of food, but not water, by transferring 50 flies into single vials containing $2 \mathrm{~mL}$ of $0.5 \%$ agar for $12 \mathrm{~h}$ preceding a given trial. If an experiment included food, we placed a small patch of food (identical to that used for rearing) on top of a $2-\mathrm{mL}$ plug of $0.5 \%$ agar that was embedded into the center of the floor within each chamber. All chambers provided access to similar plugs of agar to prevent dehydration. We introduced flies into chambers at 09:00 and waited $1 \mathrm{~h}$ for the flies to settle down before starting each trial. We programmed the solenoid-controlled gates to open precisely at 10:00 and monitored the movements of flies until 16:00. In these and all subsequent experiments, we ran trials during a $6 \mathrm{~h}$ time window in the middle of the subjective day to avoid confounding interactions with the morning and evening peaks in activity.

After approximately $4 \mathrm{~h}$, the dispersal rate of the flies reached a state of equilibrium between chambers that both contained only water (see Fig. 3 for sample data, equilibrium denoted by arrowhead in c). The flies reached an equilibrium sooner if we decreased the length of the

Table 2 Ambient environmental conditions from experiments within this study and from a representative sample of studies published from the 1970s until present on the behavior of Drosophila melanogaster

\begin{tabular}{llllll}
\hline Experiment & Figure(s) & Year & Duration (days) & ${\text { Temperature }\left({ }^{\circ} \mathrm{C}\right)^{\mathrm{a}}}^{\%_{\text {R Relative humidity }}}{ }^{\mathrm{a}}$ \\
\hline Sample data & $3 \mathrm{a}-\mathrm{f}$ & 2005 & 10 & $25.7 \pm 0.3$ & $43.3 \pm 2.7$ \\
Cross traffic & $3 \mathrm{e}-\mathrm{f}$ & 2006 & 3 & $26.4 \pm 0.2$ & $63.1 \pm 2.3$ \\
Proximity to food & Materials and methods & 2005 & 3 & $26.1 \pm 0.3$ & $60.7 \pm 5.8$ \\
Mating history & $4,5,6,7$ & 2005 & 8 & $26.1 \pm 0.5$ & $60.8 \pm 3.0$ \\
Single flies & Results & 2008 & 21 & $21.2 \pm 1.0$ & $38.0 \pm 5.7$ \\
Activity, weight & 8 & 2007 & 3 & $24.5 \pm 0.2$ & $29.2 \pm 3.5$ \\
Activity, mating & Results & 2007 & 5 & $25.6 \pm 0.3$ & $34.0 \pm 0.9$ \\
Genetic background & 9 & 2004 & 16 & $\approx 25^{\mathrm{b}}$ & $\mathrm{n} . \mathrm{r}$ \\
Foraging gene & 10 & 2005 & 10 & $25.7 \pm 0.3$ & $43.3 \pm 2.7$ \\
Literature & & $>1970$ & & $24.1 \pm 1.8$ & $62.8 \pm 9.4$ \\
\hline
\end{tabular}

n.r. Not recorded

${ }^{\mathrm{a}}$ Mean $\pm \mathrm{STD},{ }^{\mathrm{b}}$ Incubator, ${ }^{\mathrm{c}}$ From 62 articles 

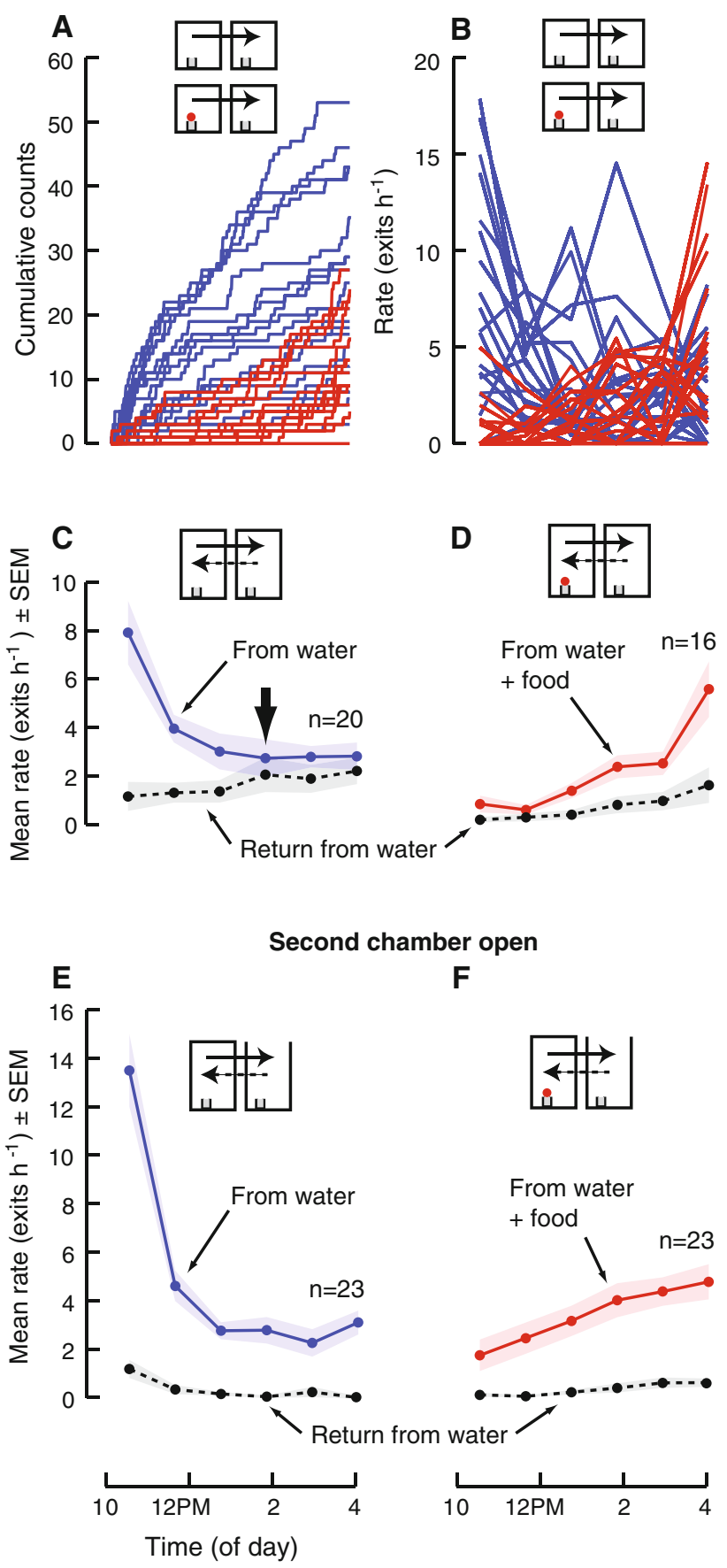

channel connecting the chambers or widened the diameter of the exit hole leading into this channel (Simon 2010). Conversely, if we increased the length of the channel or narrowed the diameter of the exit hole leading into the channel, we could lengthen the time required to reach equilibrium (Simon 2010). We chose a length of channel connecting the chambers as well as a width of exit hole leading into this channel that would minimize instances of cross traffic.
Fig. 3 Sample data showing the movement of flies between two connected chambers containing either a patch of food or only water. a, b Cumulative forward counts and corresponding forward rates from independent trials for transitions from water (blue, $2 \mathrm{~mL} 0.5 \%$ agar) and a patch of food ( $\mathrm{red}, 65 \mu \mathrm{L}$ on $2 \mathrm{~mL}$ of $0.5 \%$ agar) contained in a first chamber to a second chamber containing water. c Means \pm SEM for forward (blue solid) and reverse (black dashed) rates each hour from trials where both chambers contained water. Flies reached equilibrium movement between chambers in this particular experiment after $4 \mathrm{~h}$ (arrowhead). d Means \pm SEM for forward (red solid) and reverse (black dashed) rates from trials where the first chambers contained a patch of food and the second chamber contained water. e, f Flies introduced to the first of two connected chambers moved comparably from the first to a second chamber whether or not the lid to the second chamber was closed or open (compare $\mathbf{c}, \mathbf{d}$ to $\mathbf{e}, \mathbf{f}$ )

To demonstrate that the level of cross traffic for the chosen channel length and exit hole width would not appreciably interfere with our measurements of the flies' movement, we ran experiments similar to those described in Fig. 3a-d, but modified the experiments by removing the lid covering the second chamber (Fig. 3e, f), allowing the flies to escape into the room. Removing the lid of the second chamber increased the level of dispersal (ANOVA, lid condition, $P<0.05$ ), but did not alter the relative difference in dispersal from chambers containing food or water (Fig. 3; compare c, d to e, f; ANOVA, interaction between food and lid condition, $P=0.856$ ).

Flies that had moved to the second chamber returned to the first chamber at a similar rate irrespective of the presence of food in the first chamber. (For an example, see Fig. 3c, d; Mann-Whitney $U, P=0.147$.) Also, the mean rates for flies dispersing from water towards chambers containing only water $\left(9.4 \pm 1.3\right.$ exit $\left.^{-1}, n=14\right), 65 \mu \mathrm{L}$ of food and water $\left(10.3 \pm 0.9\right.$ exit $\left.\mathrm{h}^{-1}, n=14\right)$, and $100 \mu \mathrm{L}$ of food and water $\left(10.1 \pm 0.9\right.$ exit $\left.^{-1}, n=15\right)$ were similar over the first hour (ANOVA, $P=0.816$ ). This indicates that the presence of food in a chamber did not attract flies from adjoining chambers. However, once flies found food in the second chamber, their return to the adjoining chamber was inhibited. Compared to water alone, both $65-$ and $100-\mu \mathrm{L}$ patches of food inhibited the return movement of the flies $(65 \mu \mathrm{L}$, Mann-Whitney $U$, $P=0.002 ; 100 \mu \mathrm{L}$, Mann-Whitney $U, P<0.0001)$. From these observations we conclude that the flies could not perceive food in adjoining chambers and responded only to food in the chamber they were in. For all experiments, we ran simultaneous trials in 16 pairs of connected chambers. Within a given experiment, we pooled results from trials run over several days. Unless otherwise indicated, all data are reported as mean \pm SEM exit rates per hour, were averaged over $6 \mathrm{~h}$ for statistical analyses, and when appropriate were corrected (Bonferroni corrections are noted throughout the text as ${ }^{B F}$; SPSS, SPSS, Inc., Chicago, Illinois, USA). 


\section{Activity experiments}

To test whether a change in the intensity of a fly's general activity might have contributed to the differences we observed in their dispersal, we measured the effects of sex, weight, and mating status on their general locomotor activity using Drosophila Activity Monitors (DAM2, TriKinetics, Inc., Waltham, Massachusetts, USA). Unless otherwise specified, we reared, housed, entrained, and handled flies, as well as ran experiments over the same midday, 6-h time window, as in the dispersal experiments. After weighing flies, we placed them for $12 \mathrm{~h}$ into separate vials containing food. The following day, after allowing flies to settle for $1 \mathrm{~h}$, we measured their activity using the TriKinetics monitors. Unless noted, flies were sated at the start of each trial, and during trials had access only to water. When the signal from a monitor for a particular fly stopped registering events, and continued not registering events throughout the rest of the experiment, we assumed that this indicated the death of the fly. We adjusted the calculation for mean activity for each 5-min period throughout the experiment, taking into account the death of any individuals during the trial period.

\section{Results}

To examine sex differences in dispersal, we introduced groups composed of only males or only females into chambers with either food or water and monitored their dispersal. The most obvious result is that mated males dispersed from water at a much higher rate than mated

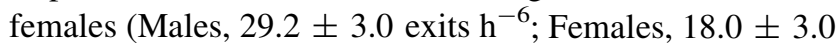
exits $\mathrm{h}^{-6}$; Fig. $4 \mathrm{a}, \mathrm{b}$ and Fig. $5 \mathrm{a}-\mathrm{c}$; ${ }^{i} t$-test, $P=0.036^{B F}$ ). This was not an emergent property of being in a group. When tested individually, single mated males also dispersed from water at a higher rate than single mated females (Males, $n=43$; Females, $n=51$; Mann-Whitney $U, P=0.023)$.

The second result to note is that the presence of food drastically reduced the dispersal of both mated males and females. (Fig. 4a, All-male group, Mann-Whitney $U$, $P<0.0001^{B F} ; \mathrm{b}$, For all-female group, inhibition was statistically significant for only the first $3 \mathrm{~h}$ : first and second, Mann-Whitney $U, P<0.0001^{B F} ; P<0.0001^{B F}$; third, $t$-test, $\left.P=0.028^{B F}\right)$. Again, this result was true whether the flies were tested in groups or as single individuals. Single flies never left chambers containing food during $12 \mathrm{~h}$ of observation (12 males and 12 females). Because chambers containing food also contained water, the influence of food itself is best estimated by subtracting the dispersal rate from food from the dispersal rate from water (Fig. 4c). This difference is larger for mated males than mated females ( $i$ one-sample $t$-test, $P<0.0001$ ), presumably because in the absence of food mated males exhibit such a high dispersal rate.

To test the influence of mating status on dispersal from both food and water, we compared the dispersal of both males and females with prior mating experience to that of virgin flies. Mating had a very strong effect on the dispersal of flies from water, particularly in males (Fig. 5a-d; ${ }^{i i} \mathrm{All}-$ male group, Mann-Whitney $U, P=0.009^{B F} ;{ }^{i i i}$ All-female
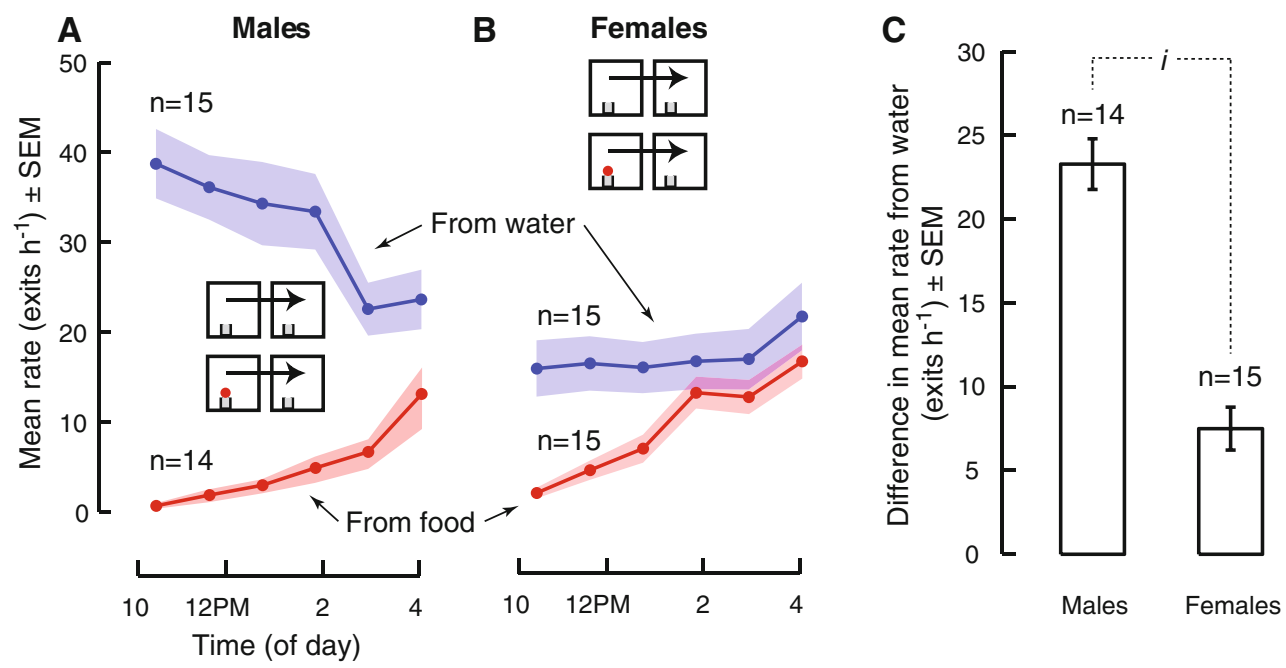

Fig. 4 Food inhibits the dispersal of mated flies to a greater extent for males relative to females. a, b Food ( $r e d, 65 \mu \mathrm{L}$ on $2 \mathrm{~mL} 0.5 \%$ agar) placed in model environments inhibits the dispersal of both male and female flies with previous mating experience relative to dispersal from only water (blue, $2 \mathrm{~mL} 0.5 \%$ agar). c The difference in dispersal between from food and the collective mean rate from water was greater for mated males than the difference for mated females $(i)$. Mean \pm SEM differences between the dispersal from food and the collective mean average hourly rates from water over $6 \mathrm{~h}$ (Males, 29.2 exits $\mathrm{h}^{-1}$; Females, 18.0 exits $\mathrm{h}^{-1}$ ) 


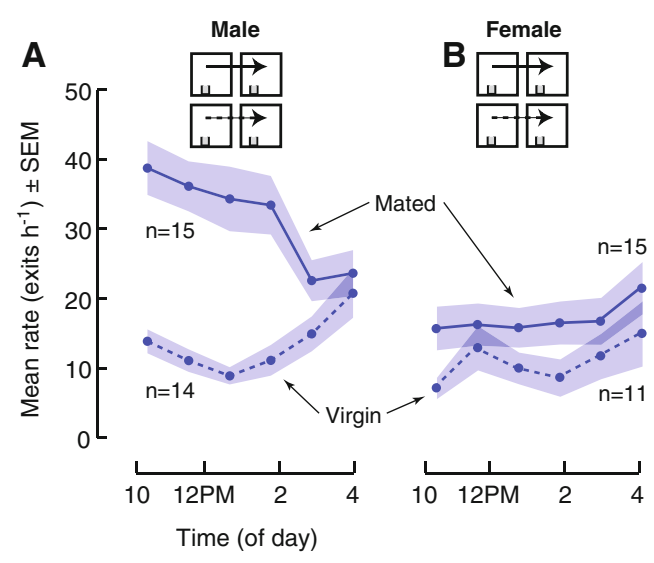

Fig. 5 Mating increases the dispersal of flies from water to a greater extent for males relative to females. a, b From model environments containing only water ( $2 \mathrm{~mL} 0.5 \%$ agar), groups of previously mated (solid) males and females dispersed at a higher rate than virgin (dashed) males and females. c Mated males dispersed from water at a

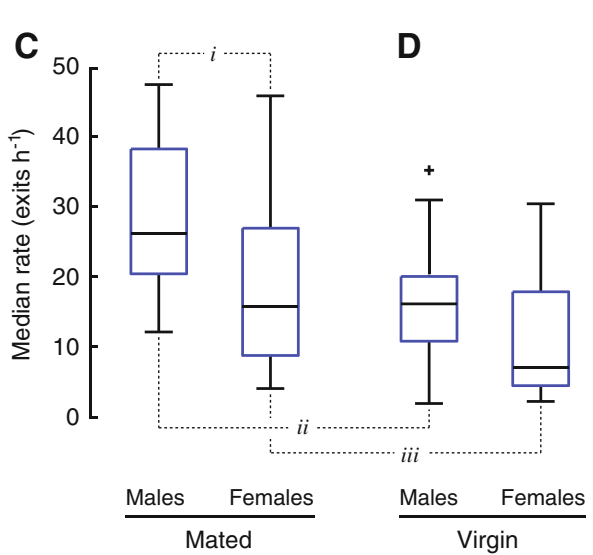

higher rate than mated females $(i)$. c, $\mathbf{d}$ Mating increased the dispersal of males (ii) and females (iii) from water. Median hourly rates over 6 $\mathrm{h}$. The top and bottom edges of the boxes represent 75 th and 25th percentiles; the whiskers extend to the most extreme point not considered outliers, and outliers are plotted individually $(+)$
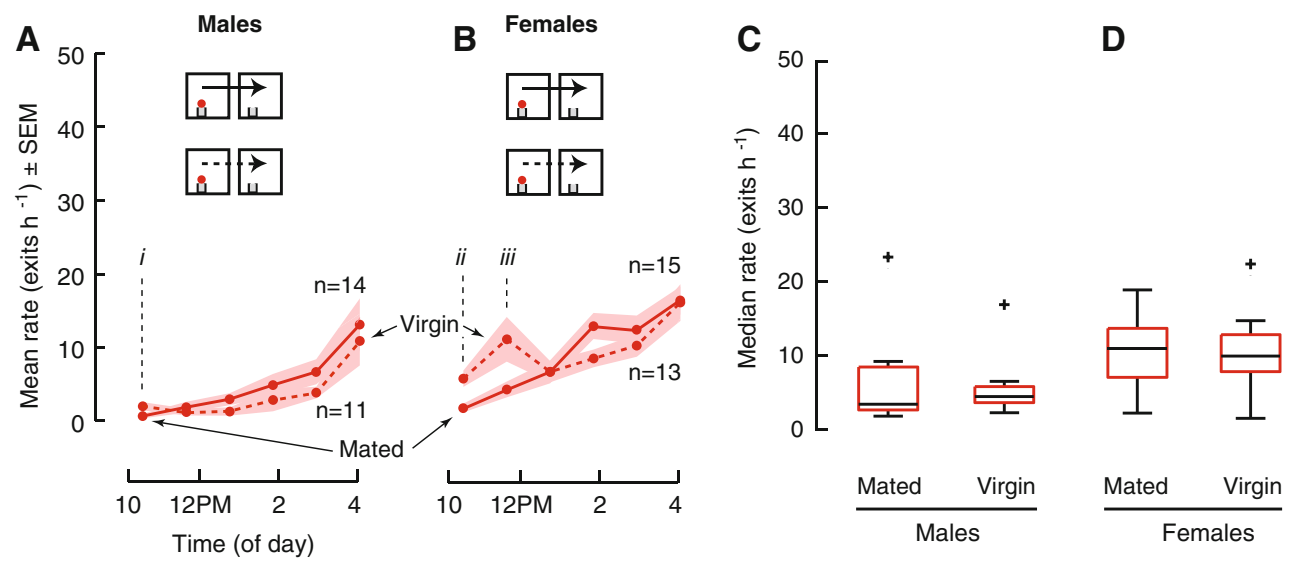

Fig. 6 Mating inhibits the immediate dispersal of flies from food. a, b From model environments containing food $(65 \mu \mathrm{L} 2 \mathrm{~mL} 0.5 \%$ agar), groups of previously mated (solid) males and females dispersed at a lower rate than virgin (dashed) males and females. Mating inhibited the dispersal of males during the first hour $(i)$ and females

group, ANOVA, $P=0.003^{B F}$ ). The influence of mating on dispersal from food (Fig. 6) was much subtler, possibly because the strong inhibitory effect of food on dispersal rate obscures any further influence of mating. After being introduced to chambers containing food, mated males dispersed at a lower rate than virgin males during the first hour (Fig. 6a; ${ }^{i}$-test, $P=0.037^{B F}$ ) and mated females dispersed at a lower rate during the first and second hours than virgin females (Fig. $6 \mathrm{~b} ;{ }^{i i}$ t-test, $P=0.015 ;{ }^{i i i}$ MannWhitney $U, P=0.058^{B F}$, 1-tailed).

The data plotted in Figs. 5 and 6 may be replotted to examine the influence of food on the dispersal of virgins, just as Fig. 4 illustrates the influence of food on mated flies. The data indicate that the presence of food inhibits the dispersal of virgin males (Fig. $7 \mathrm{a}, \mathrm{c} ;{ }^{i} t$-test, $P=0.003^{B F}$ ), during the first $2 \mathrm{~h}$ (ii, iii) from food, but not thereafter. c, d Median hourly rates over $6 \mathrm{~h}$. The top and bottom edges of the boxes represent 75th and 25th percentiles; the whiskers extend to the most extreme point not considered outliers, and outliers are plotted individually $(+)$

so that the resulting rate is lower than that for virgin females (Fig. $7 \mathrm{a}-\mathrm{d}$; ${ }^{i i} t$-test, $P=0.045^{B F}$ ), whereas food does not alter the dispersal rate of virgin females (Fig. 7b, d; Mann-Whitney $U, P=0.543$ ).

We reported above that mated males dispersed at a higher rate than mated females from chambers containing only water. One possible explanation for this observation is that it represents a fundamental difference in the levels of general activity rather than a difference in locomotory behaviors related to dispersal per se. This is not an easy distinction to make based on any locomotor assay, but to gain some additional insight we introduced individual flies fed ad libitum into simple beam crossing activity monitors and measured their behavior until all flies had died from starvation. We found that males did exhibit a higher level 

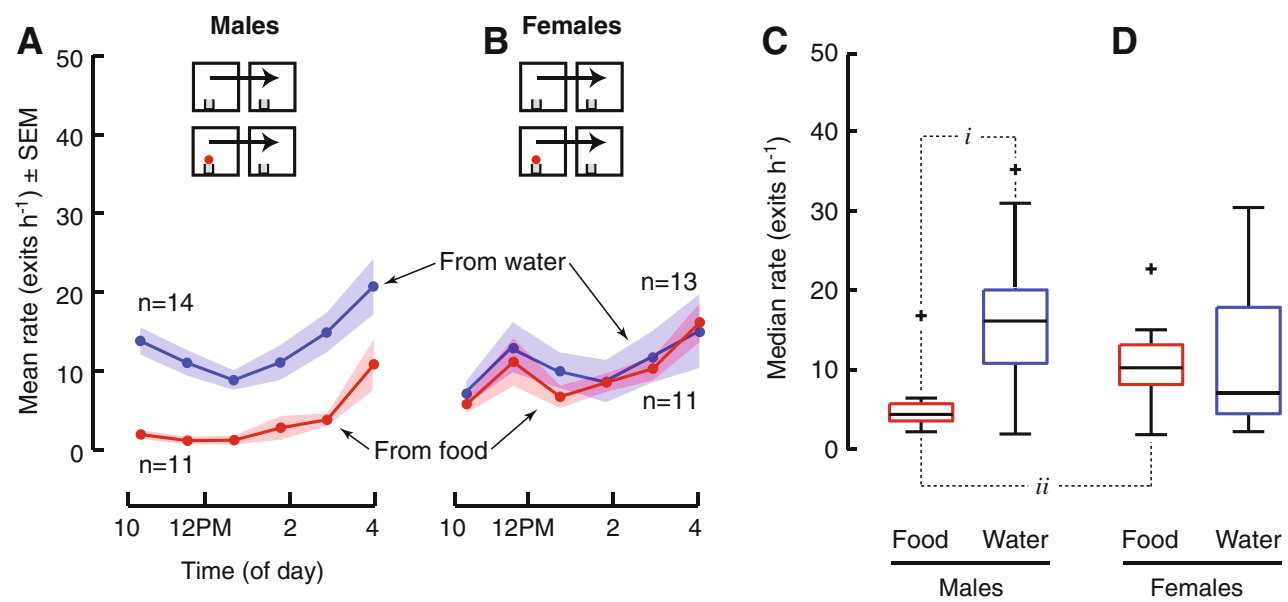

Fig. 7 Virgin females disperse similarly from food or only water. a, b The presence of food (red, $65 \mu \mathrm{L}$ on $2 \mathrm{~mL} 0.5 \%$ agar) in a model environment inhibited the rate of dispersal for virgin male flies, but had no affect on the rate of dispersal for virgin females relative to dispersal from only water (blue, $2 \mathrm{~mL} 0.5 \%$ agar). c Virgin males dispersed from food at a lower rate than from water $(i)$. c, d Virgin males dispersed from food at a lower rate than virgin females (ii). Median hourly rates over $6 \mathrm{~h}$. The top and bottom edges of the boxes represent 75 th and 25 th percentiles; the whiskers extend to the most extreme point not considered outliers, and outliers are plotted individually $(+)$

activity between mated and virgin females ( $5.4 \pm 0.7$ beam crosses $\mathrm{h}^{-6}, n=22$ vs. $4.2 \pm 0.4$ beam crosses $\mathrm{h}^{-6}$, $n=23$; Wilcoxon rank sum test, $P=0.223$ ). From these experiments, we suggest that a change in general activity, as measured with widely utilized Drosophila activity monitors, cannot directly explain the influence of mating experience on dispersal as measured with our automated chambers.

Many studies on the behavior of Drosophila have been carried out using derivatives of the standard laboratory wild-type Canton-S strain (CS). We report a large difference in the dispersal behavior between our natural isolate (NI) and CS from a chamber containing food to a second chamber containing no food, and a subtle difference in dispersal from chambers containing only water (Fig. 9). The dispersal rate for NI from food was greater than 1 exit per hour by the third hour of the experiment (open diamond, one-sample $t$-test, $P=0.003$ ), but did not exceed this level until the ninth hour for CS (open square, onesample $t$-test, $P=0.048$, 1-tailed). From chambers containing only water, the dispersal rate for the NI (13.4 \pm 1.4 exit $\left.^{-1}\right)$ was higher than that from CS $(7.5 \pm 1.0$ exit $\mathrm{h}^{-1}$ ) during the first hour $(* t$-test, $P=0.001)$, but not for the remainder of the experiment. Dispersal rates during the second hour, for example, were $6.2 \pm 0.7$ exits $h^{-1}$ (NI) and $8.1 \pm 1.0$ exits $\mathrm{h}^{-1}(\mathrm{CS})$, and were not statistically different ( $t$-test, $P=0.127$ ). We chose to continue our experiments using our NI stock because it is likely to be less affected by genetic bottlenecks.

Flies possessing sitter or rover, allelic forms of the foraging gene (Osborne et al. 1997), have been shown to 
Fig. 8 A fly's sex and not weight explains why males are more active than females. We measured the activity of individual flies using the commercially available Drosophila Activity Monitor (DAM2). a Male flies (blue) exhibited a greater mean activity level than females (red) during the same time window as the experiments on dispersal carried out in this study (horizontal black bar). b During the fourth hour (vertical green line in a), males were

approximately twice as active as females. c Females weighed nearly twice that of males. d By comparing the activity and weight of individual males (open circles) and females (closed circles) we show that the contribution of weight to the activity of flies was minimal and insignificant. By adjusting the measures of activity for weight (dashed lines near respective mean activity), we illustrate that the sex of a fly contributes to why males are more active than females

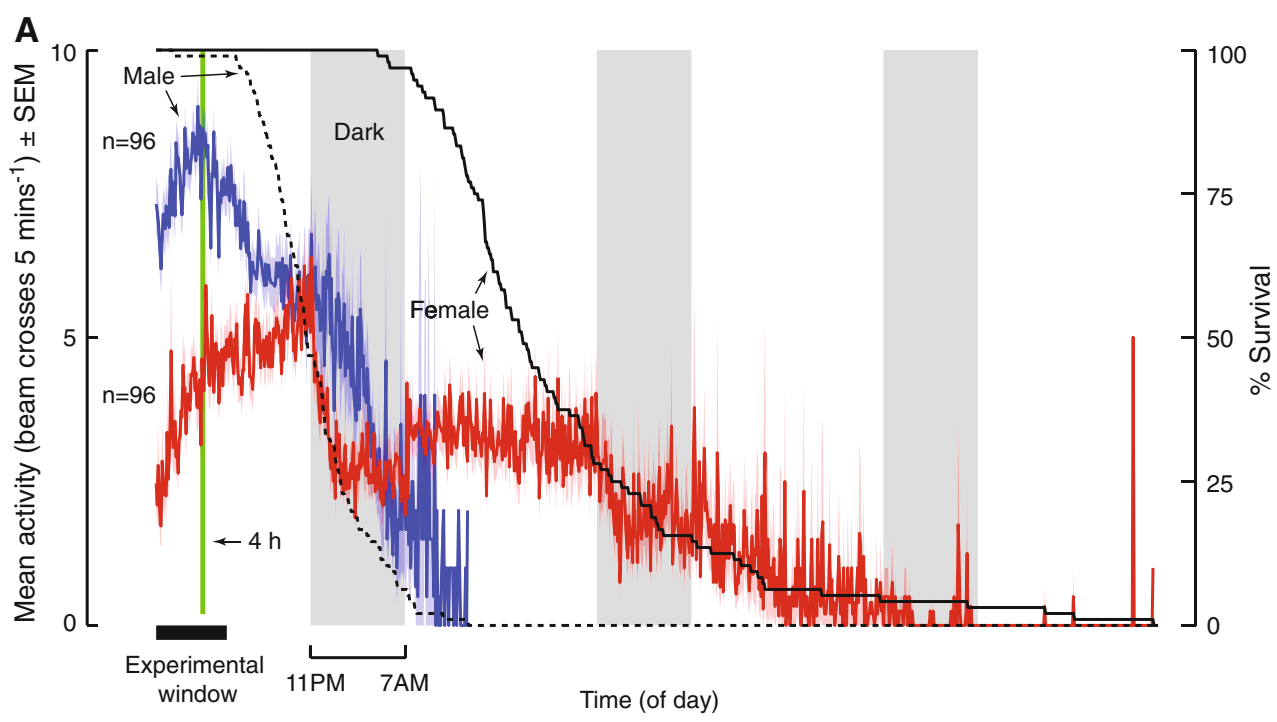

C

B

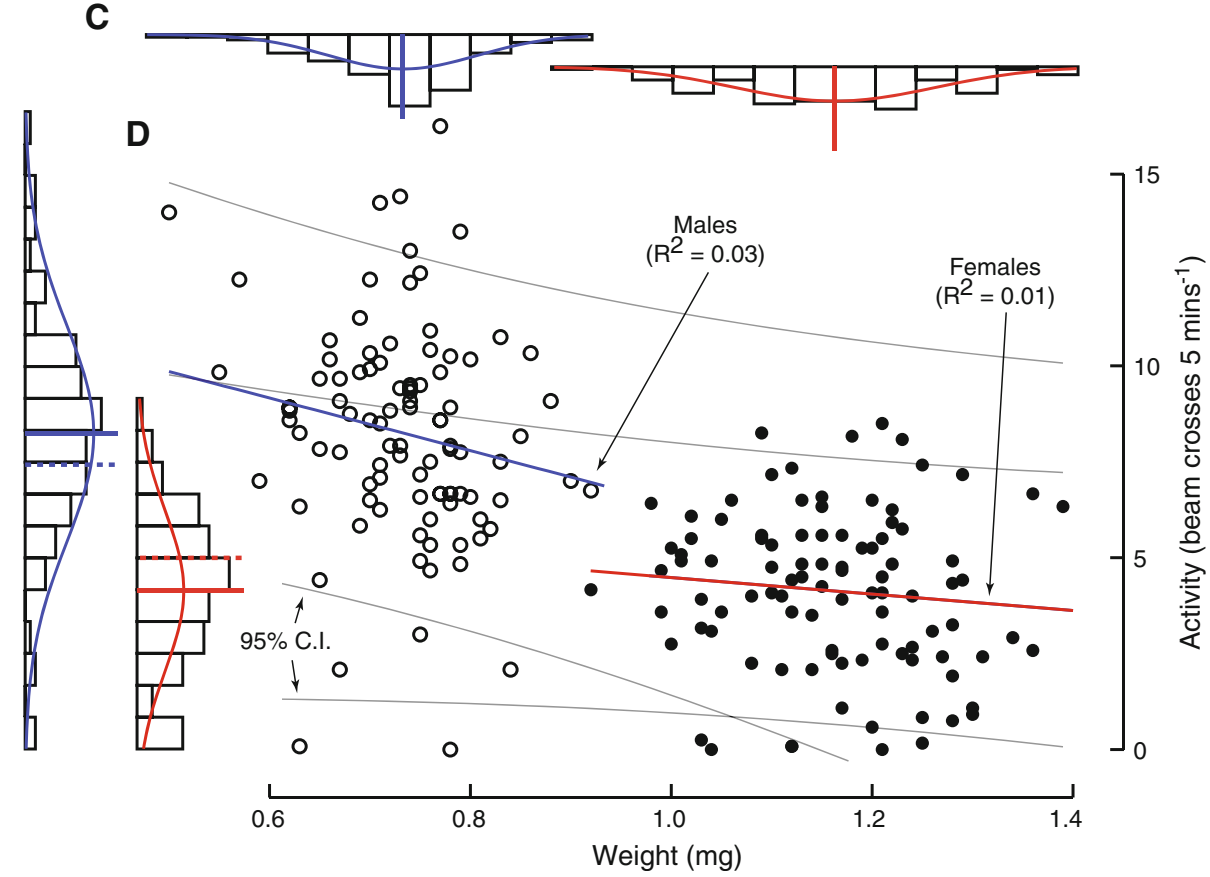

exhibit significant differences in their movement on and around food (Pereira and Sokolowski 1993). Therefore, one possible explanation for the large difference in dispersal that we observed between the NI and the CS strains is that the sitter allele has been selected for over the last 80 years and has become fixed in the CS stock used within this study (or vice versa the rover allele has come to dominate the NI). This does not appear to be the case, however, for we observed flies homogeneous for sitter or rover to disperse at comparable rates from food (Fig. 10). Flies with the naturally occurring sitter polymorphism exhibited a sometimes similar, but overall higher rate of dispersal from food $\left(3.1 \pm 0.4\right.$ exit $\left.\mathrm{h}^{-6}, n=16\right)$ than rover $(1.5 \pm$ 0.4 exit $\left.\mathrm{h}^{-6}, n=15\right)(t$-test, $P=0.003)$. Consistent with a non-significant trend reported previously (Pereira and Sokolowski 1993), we observed that flies with the sitter polymorphism dispersed at a lower rate (Forward, $4.9 \pm$ 0.5 exit $\mathrm{h}^{-6}$, Return, $2.9 \pm 0.4$ exit $\mathrm{h}^{-6}, n=20$ ) between chambers containing only water than rover (Forward, $7.9 \pm 0.9$ exit $\mathrm{h}^{-6}$, Return, $4.9 \pm 0.8$ exit $^{-6}, n=20$ ) (Forward, $t$-test, $P=0.005$; Return, $t$-test, $P=0.038$ ).

\section{Discussion}

The act of mating changes the resources required for an individual (Markow et al. 1999), potentially making the decision to stay on a resource or to leave in search of 


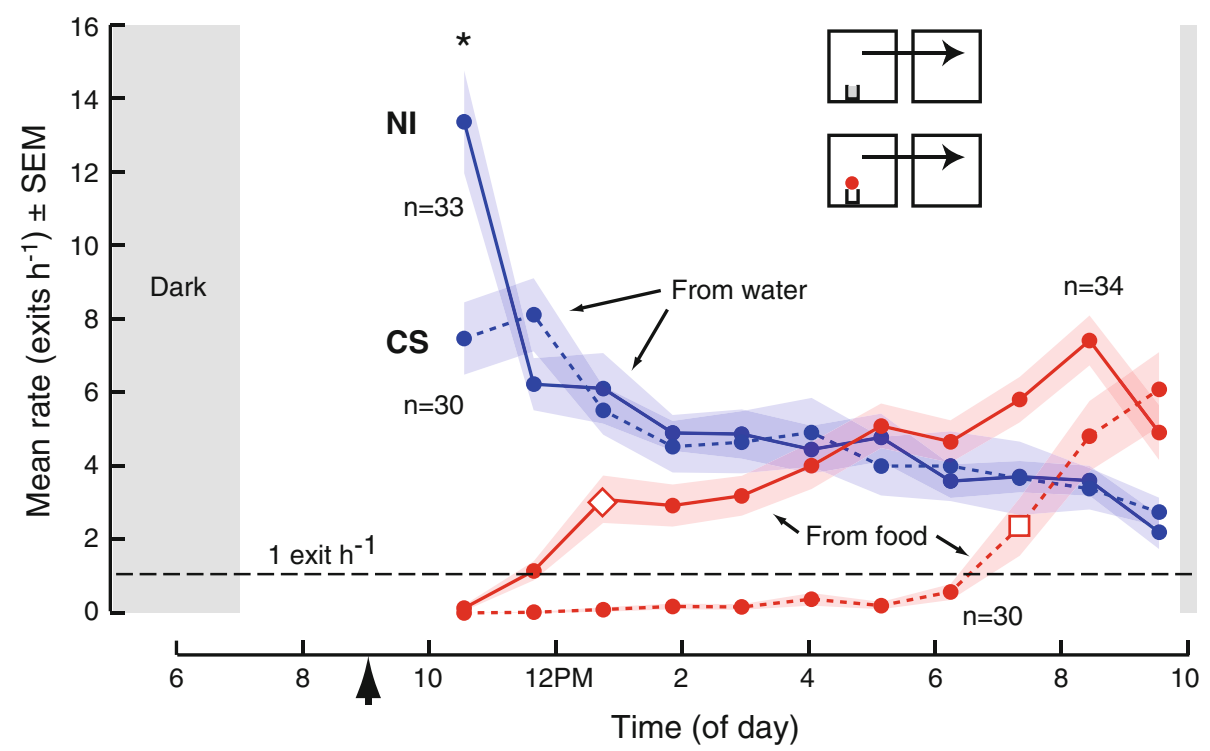

Fig. 9 Genetic background is a significant factor in the dispersal of Drosophila from food. The figure shows the dispersal rates of the natural isolate (NI, solid) used throughout this report and Canton-S (CS, dashed) from chambers that contained a patch of food (red, $65 \mu \mathrm{L}$ on $2 \mathrm{~mL}$ of $0.5 \%$ agar) and chambers containing only water (blue, $2 \mathrm{~mL}$ of $0.5 \%$ agar). From food, the mean dispersal rates were greater than 1 exit per hour for the NI by the third hour (open

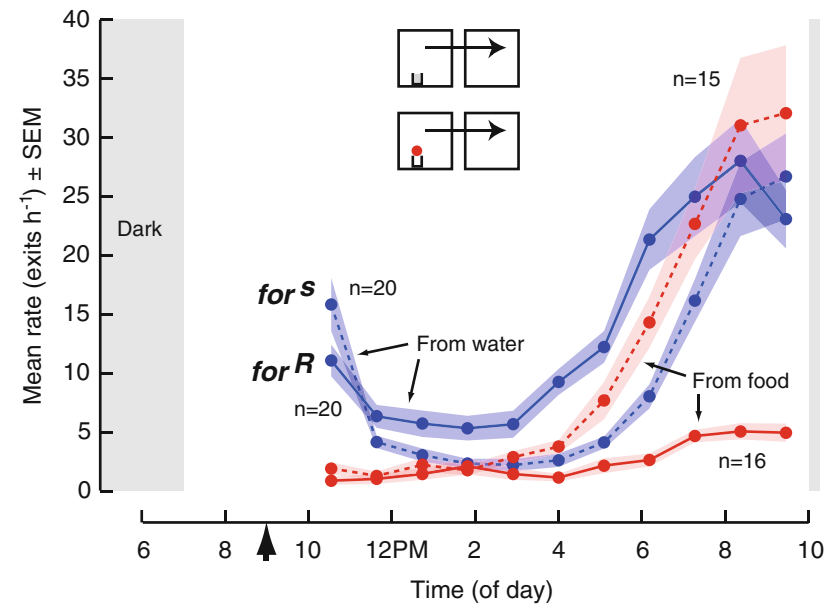

Fig. 10 Allelic fixation cannot explain the difference in dispersal observed between the natural isolate and laboratory strain used within this report. The figure shows the dispersal rates of flies possessing the naturally occurring polymorphic alleles that are known for restricted $\left(\right.$ for $^{S}$, dashed) and active (for ${ }^{R}$, solid) searching behavior near food, from chambers that contained a patch of food (red, $65 \mu \mathrm{L}$ on $2 \mathrm{~mL}$ of $0.5 \%$ agar) and chambers containing only water (blue, $2 \mathrm{~mL}$ of $0.5 \%$ agar). Over the first $6 \mathrm{~h}$, flies dispersed at a comparable rate irrespective the allele they possessed (see text for details). The gray rectangles indicate subjective dawn and dusk. We started experiments $1 \mathrm{~h}$ after introducing flies (arrowhead) into chambers, allowing the flies time to settle

superior resources critically important. We therefore set out to investigate the influence of a fly's prior mating experience on its dispersal. Based on a laboratory assay, we diamond) and did not exceed this level until the ninth hour for CS (open square). For the first hour, groups of the NI dispersed at a higher rate than groups of CS from water (asterisks). The gray rectangles indicate subjective dawn and dusk. We started experiments $1 \mathrm{~h}$ after introducing flies (arrowhead) into chambers, allowing the flies time to settle

suggest that prior mating experience enhances the dispersal of both male and female Drosophila, increasing the dispersal of males to a greater extent than females. In contrast, if we introduced flies into environments containing food, males dispersed at a lower rate than females, with prior mating experience further inhibiting dispersal. For males, the increase in dispersal may be due to a change in the general level of their activity. However, for females the increase in dispersal appears to be independent of a change in their level of activity. In general, flies with mating experience behave as if they are hungrier than virgin flies, leaving at a greater rate when food is unavailable and staying longer when it is available.

Laboratory studies of the dispersive movements of Drosophila are not new. After the development of a series of connected chambers by Sakai and colleagues (Sakai et al. 1958), many studies have been carried out within a laboratory setting attempting to identify the various abiotic and biotic factors contributing to the movement patterns of Drosophila (for a review see (Grossfield 1978)). For the studies carried out within this report we have built we have built an automated modular system akin to the simple system developed by Sakai and colleagues (Sakai et al. 1958), with the added features of being able to regulate and continually quantify the back and forth movements of flies among distinct model environments without disruption.

The high level of variability seen in laboratory and field studies on the sexual dimorphism of Drosophila migration 
(see (Iliadi et al. 2002)) presumably reflects the experimental conditions of each particular study, might be specific to the particular species, and is likely to be attributed to the inherent breeding and nutritional needs required by each sex. For resource specialists, such as the cactophilic Drosophila, it has been shown that the differences in dispersal among various species is related to the spatial distribution and the predictability of the specific host patches utilized by each species (Markow and Castrezana 2000). For generalists, such as the "cosmopolitan" Drosophila that are found to coexist world-wide, these species show a consistent species-specific progression of colonization that is driven by the state of microbial decay of a resource (Nunney 1990, Nunney 1996). Inorganic nutrient availability has been shown to limit fertility (Sang 1978) and field studies measuring the ratio of phosphorus to nitrogen found in females and males support the hypothesis that females may seek resources containing phosphorus more than males (Markow et al. 1999). It is therefore reasonable to believe that the breeding site selected by females is governed by the nutrient content of a resource (Jaenike and Markow 2003). In addition to the specific nutrient contents required by gametogenesis (Markow et al. 1999), the amount (Magwere et al. 2004) and type (Andersen et al. 2010) of nutrients required by females has also been shown to differ from those required by males for supporting various life history traits (e.g., tolerance to stress, longevity, reproduction). Whereas we have yet to determine the specific components of a resource that inhibit the dispersal of males, our observation that males dispersed from food at a lower rate than females is consistent with previous findings for natural isolates tested at the optimum temperature for these flies (Iliadi et al. 2002; Mikasa and Narise 1980) (however, see Mckenzie 1974; Mikasa 1992).

The results from our activity studies indicate that the greater dispersal observed in males may reflect an intrinsic difference in the locomotor activity between males and females. Males exhibiting a higher level of activity than females has been shown before, first observing individuals over the period of minutes (Burnet et al. 1988), then hours (Martin 2004) and more recently in a social context (Branson et al. 2009). We add to this prior knowledge that the difference in activity cannot merely be explained by the disparity among the weight of flies, and suggest that the differences we observed in dispersal result from intrinsic differences between males and females of this species (however, see Belgacem and Martin (2007)). Results from measuring the general locomotor activity of mated and virgin flies suggest that a change in the general locomotor activity alone was insufficient to explain the effect of mating on the dispersal of females, and further indicate that the influence of activity on dispersal of males is dependent on their mating status. The discrepancy between our findings on the activity of mated females and those reported by Isaac et al. (2010) presumably reflects that they tested females taken directly off food and given access to $5 \%$ sugar throughout their activity trials.

Variation in dispersal within a population is most probably due to the interaction between genetic and environmental causes (Dingle 1991) making it difficult to attribute a causative role for either. The system of experimental chambers described within this study provides a method for parallel "common garden" experiments where we can hold constant specified abiotic conditions with the hope of quantifying the genetic components contributing to the variation. In side-by-side experiments, we observed a considerable difference in the dispersal between the Canton-S strain and the natural isolate used throughout this study. This observation was not surprising, for it has been shown previously that laboratory strains exhibit lower levels of dispersal than wild strains (Mikasa and Narise 1980; Tantawy et al. 1975). A possible explanation for this striking variability is that the NI and the CS stocks exhibit allelic partitioning at the foraging locus, with the genetic constitution of the NI being entirely, or mostly, that of the rover allele and the CS stock the sitter allele. This explanation seems unlikely for we observed comparable dispersal between flies homogeneous for these polymorphic alleles. This experiment does, however, illustrate the feasibility of this line of study, provides evidence that these types of experiments may help to clarify the relative contribution of genes and environment for this trait, and should uncover additional unexpected findings such as the striking increase in the dispersal of flies possessing the sitter allele from food as they near subjective dusk (see Fig. 10). Finally, the magnitude of the difference between CS and our NI raises concern when evaluating studies carried out with Canton$S$ (or any stocks derived from this stock) and perhaps any stock cultivated too long within the laboratory.

From these studies, we suggest that prior mating experience is a significant and likely important factor modulating the dispersal of Drosophila. We believe we are the first to demonstrate an influence of prior mating experience on the dispersal of males, and as well, to directly compare their dispersal to that of females from distinct environments with and without food. Variability in dispersal is a significant factor driving the change of genetic structure within a population, however, a richer description of dispersal is required before making conclusions regarding the mechanisms underlying the various factors contributing to this complex behavior. It would be informative to directly observe both the movement of single flies and individual flies behaving within groups as they disperse from patches of food. This is a direction of research that we are currently pursuing. 
Acknowledgments We thank R. Bailey for help in developing the circuit boards that run our technology; J. Birch for early drafts of the machine drawings used in the design of our hardware; M. Vondrus for help with machining various parts used in the construction of our experimental chambers; M. Sokolowski for fly strains; the late S. Benzer and members of his laboratory for much criticism; and finally, members of the Dickinson laboratory for experimental guidance and helpful discussions. This work was supported by NIH grant 5R01DA22777-3, NSF Engineering Research Center Grant EEC9407 226, and HHMI (J.C.S.).

Open Access This article is distributed under the terms of the Creative Commons Attribution Noncommercial License which permits any noncommercial use, distribution, and reproduction in any medium, provided the original author(s) and source are credited.

\section{References}

Alyokhin AV, Ferro DN (1999) Reproduction and dispersal of summer-generation colorado potato beetle (Coleoptera: Chrysomelidae). Environ Entomol 28(3):425-430

Andersen LH, Kristensen TN, Loeschcke V, Toft S, Mayntz D (2010) Protein and carbohydrate composition of larval food affects tolerance to thermal stress and desiccation in adult Drosophila melanogaster. J Insect Physiol 56:336-340

Armes NJ, Cooter RJ (1991) Effects of age and mated status on flight potential of Helicoverpa armigera (Lepidoptera: Noctuidae). Physiol Entomol 16:131-144

Barton Browne L (1993) Physiologically induced changes in resource-oriented behavior. Annual Review of Entomology 38:1-25

Belgacem YH, Martin JR (2007) Hmger in the corpus allatum controls sexual dimorphism of locomotor activity and body size via the insulin pathway in Drosophila. PLoS ONE 2(1):e187

Bellamy DE, Byrne DN (2001) Effects of gender and mating status on self-directed dispersal by the whitefly parasitoid Eretmocerus eremicus. Ecol Entomol 26:571-577

Brady J, Gibson G (1983) Activity patterns in pregnant tsetse flies, Glossina morsitans. Physiol Entomol 8:359-369

Branson K, Robie AA, Bender J, Perona P, Dickinson MH (2009) High-throughput ethomics in large groups of Drosophila. Nat Methods 6:451-457

Burnet B, Burnet L, Connolly K, Williamson N (1988) A genetic analysis of locomotor activity in Drosophila melanogaster. Heredity 61:111-119

Callaway EM (2005) A molecular and genetic arsenal for systems neuroscience. Trends Neurosci 28:196-201

Carvalho GB, Kapahi P, Anderson DJ, Benzer S (2006) Allocrine modulation of feeding behavior by the sex peptide of Drosophila. Curr Biol 16:692-6

Cornelius ML, Nergel L, Daun JJ, Messing RH (2000) Reponses of female oriental fruit flies (Diptera: Tephritidae) to protein and host fruit odors in field cage and open field tests. Environ Entomol 29(1):14-19

Dethier V (1964) Microscopic brains. Science 143:1138-1145

Dingle H (1966) Some factors affecting flight activity in individual milkweed bugs (oncopeltus). J Exp Biol 44:335-343

Dingle H (1991) Evolutionary genetics of animal migration. Am Zool 31:253-264

Dobzhansky T (1973) Active dispersal and passive transport in Drosophila. Evolution 27(556-575)

Feder M (1997) Necrotic fruit: a novel model system for thermal ecologist. J Thermal Biol 22(1):1-9
Green GW (1962) Flight and dispersal of the european pine shoot moth, Rhyacionia buoliana (schiff.) i. factors affecting flight, and the flight potential of females. Can Entomol 94:282-299

Grossfield J (1978) Non-sexual behavior of Drosophila. In: Ashburner M, Wright T (eds) The genetics and biology of Drosophila, vol 2b. Academic Press, London, pp 67-74

Iliadi KG, Iliadi NN, Rashkovetsky EL, Girin SV, Nevo E, Korol AB (2002) Sexual differences for emigration behavior in natural populations of Drosophila melanogaster. Behavior Genetics 32:173-180

Isaac RE, Li C, Leedale AE, Shirras AD (2010) Drosophila male sex peptide inhibits siesta sleep and promotes locomotor activity in the post-mated female. Proc Biol Sci 277(1678):65-70

Iwanaga-Sawabe K, Kanda T (1990) Studies on the effective attractants of anopheline mosquitoes i. blood-feeding, mating and flight activity of female Anopheles balabacensis (Diptera: Culicidae). Appl Ent Zool 25(2):231-238

Jaenike J, Markow TA (2003) Comparative elemental stoichiometry of ecologically diverse Drosophila. Functional Ecology 17: $115-120$

Jang E, McInnis D, Lance D, Carvalho L (1998) Mating-induced changes in olfactory-mediated behavior of laboratory-reared normal, sterile, and wild female Mediterranean fruit flies (Diptera: Tephritidae) mated to conspecific males. Ann Entomol Soc Am 91(1):139-144

Jang E, McInnis D, Kurashima R, Carvalho L (1999) Behavioural switch of female Mediterranean fruit fly, Ceratits capitata: mating and oviposition activity in outdoor field cages in hawaii. Agric For Entomol 1(3):179-184

Johnson C (1969) Migration and dispersal of insects by flight. Methuen \& Co., London

Jones MDR (1981) The programming of circadian flight-activity in relation to mating and the gonotrophic cycle in the mosquito, Aedes aegypti. Physiol Entomol 6:307-313

Jones MDR, Gubbins SJ (1978) Changes in the circadian flight activity of the mosquito Anopheles gambiae in relation to insemination, feeding and oviposition. Physiol Entomol 3: 213-220

Kennedy J (1978) The concepts of olfactory "arrestment" and "attraction". Physiol Entomol 3:91-98

King B (1993) Flight activity in the parasitiod wasp Nasonia vitripennis (Hymenoptera: Pteromalidae). J Insect Behav 6(3): 313-321

King BH, Grimm KM, Reno HE (2000) Effects of mating on female locomotor activity in the parasitoid wasp Nasonia vitripennis (Hymenoptera: Pteromalidae). Environ Entomol 29(5):927-933

Landolt P, Heath R (1988) Effects of age, mating, and time of day on behavioral responses of female papaya fruit fly, Toxotrypana curvicauda gerstaecker (Diptera: Tephritidae), to synthetic sex pheromone. Environ Entomol 17(1):47-51

Lewis E (1960) A standard new food medium. Drosoph Inf Serv 34: $117-118$

Luo L, Callaway EM, Svoboda K (2008) Genetic dissection of neural circuits. Neuron 57(5):634-60

Magwere T, Chapman T, Partridge L (2004) Sex differences in the effect of dietary restriction on life span and mortality rates in females and male Drosophila melanogaster. J Gerontol Biol Sci 59A:3-9

Markow TA, Castrezana S (2000) Dispersal in cactophilic Drosohpila. Oikos 89:378-386

Markow TA, Raphael B, Dobberfuhl D, Breitmeyer CM, Elser JJ, Pfeiler E (1999) Elemental stoichiometry of Drosophila and their hosts. Funct Ecol 13:78-84

Martin JR (2004) A portrait of locomotor behaviour in Drosophila determined by a video-tracking paradigm. Behav Process 67:207-219 
Mckenzie J (1974) The distribution of vineyard populations of Drosophila melanogaster and Drosophila simulants during vintage and non-vintage periods. Oecologia 15:1-16

Mikasa K (1992) Quantitative genetic study on sexual difference in emigration behavior of Drosophila melanogaster in a natural population. Jpn J Genet 67:463-472

Mikasa K (1998) Intraspecific variation in the effects of mating on emigration activity and fecundity in a natural population of Drosophila melanogaster. Genes Genet Syst 73:263-269

Mikasa K, Narise T (1980) The relation between dispersive behavior and temperature in Drosophila melanogaster. ii. sex difference. Res Notes DIS 55:111-112

Nunney L (1990) Drosophila on oranges: colonization, compeition, and coexistence. Ecology 71:1904-1915

Nunney L (1996) The colonization of oranges by the cosmopolitan Drosophila. Oecologia 108:552-561

Osborne KA, Robichon A, Burgess E, Butland S, Shaw R, Coulthard A, Pereira H, Greenspan R, Sokolowski M (1997) Natural behavior polymorphism due to a cGMP-dependent protein kinase of Drosophila. Science 277:834-836

Pereira HS, Sokolowski MB (1993) Mutations in the larval foraging gene affect adult locomotory behavior after feeding in Drosophila melanogaster. Proc Natl Acad Sci 90:5044-5046

Pompanon F, Fouillet P, Bouletreau M (1999) Physiological and genetic factors as sources of variation in locomotion and activity rhythm in a parasitoid wasp (Trichogramma brassicae). Physiol Entomol 24:346-357

Ribeiro C, Dickson BJ (2010) Sex peptide receptor and neuronal tor/ s6k signaling modulate nutrient balancing in Drosophila. Curr Biol 20(11):1000-1005

Rowcliffe C, Finlayson LH (1982) Active and resting behaviour of virgin and pregnant females of Glossina morsitans morsitans westwood (Diptera: Glossinidae) in the laboratory. Bull Entomol Res 72:271-288

Rowland M (1989) Changes in the circadian flight activity of the mosquito Anopheles stephensi associated with insemination, blood-feeding, oviposition and nocturnal light intensity. Physiol Entomol 14:77-84

Sakai KI, Narise T, Hiraizumi Y, Iyma SY (1958) Studies on competition in plants and animals. ix. experimental studies on migration in Drosophila melanogaster. Evolution 12:93-101

Sang J (1978) The genetics and biology of Drosophila, vol 2a. The nutritional requirements of Drosophila. Academic Press, New York, pp 159-191

Sappington TW, Showers WB (1992) Reproductive maturity, mating status, and long-duration flight behavior of Agrotis ipsilon (Lepidoptera: Noctuidae) and the conceptual misuse of the oogensesis-flight syndrome by entomologists. Environ Entomol 21(4):677-688

Simon JC (2010) Behavioral analysis of exploration and dispersal in Drosophila. $\mathrm{PhD}$ thesis, California Institute of Technology

Stewart SD, Gaylor MJ (1994) Effects of age, sex, and reproductive status on flight by the tarnished plant bug (Heteroptera: Miridae). Environ Entomol 23(1):80-84

Suiter KA, Gould F (1992) Effects of mating status and age on dispersal behavior in the twospotted spider mite, Tetranychus urticae in response to fenvalerate-treated leaf surfaces. Entomol Exp Appl 62:1-8

Tantawy A, Mourad A, Abou-Youssef A (1975) Studies on natural populations of Drosophila. xvi. migration in Drosophila melanogaster in relation to genotype, temperature and population density. Egypt J Genetics Cytol 4:263-276

Throckmorton L (1975) The phylogeny, ecology, and geography of Drosophila. In: King R (eds) Handbook of genetics, vol 3. Plenum Press, New York, pp 421-469

Vargas MA, Luo N, Yamaguchi A, Kapahi P (2010) A role for s6 kinase and serotonin in postmating dietary switch and balance of nutrients in D. melanogaster. Curr Biol 20(11):1006-1011

Zhang F, Aravanis AM, Adamantidis A, de Lecea L, Deisseroth K (2007) Circuit-breakers: optical technologies for probing neural signals and systems. Nat Rev Neurosci 8:577-81 